\title{
DYRK1A haploinsufficiency causes a new recognizable syndrome with microcephaly, intellectual disability, speech impairment, and distinct facies
}

Jianling Ji ${ }^{1,2}$, Hane Lee ${ }^{1,2}$, Bob Argiropoulos ${ }^{3}$, Naghmeh Dorrani ${ }^{1,2,4}$, John Mann ${ }^{5}$, Julian A Martinez-Agosto ${ }^{2,4,6}$, Natalia Gomez-Ospina ${ }^{7}$, Natalie Gallant ${ }^{4}$, Jonathan A Bernstein ${ }^{7}$, Louanne Hudgins ${ }^{7}$, Leah Slattery ${ }^{7}$, Bertrand Isidor ${ }^{8}$, Cédric Le Caignec ${ }^{8}$, Albert David ${ }^{8}$, Ewa Obersztyn ${ }^{9}$, Barbara Wiśniowiecka-Kowalnik ${ }^{9}$, Michelle Fox ${ }^{2,4}$, Joshua L Deignan ${ }^{1,2}$, Eric Vilain ${ }^{1,2,4,6}$, Emily Hendricks ${ }^{10}$, Margaret Horton Harr ${ }^{11}$, Sarah E Noon ${ }^{11}$, Jessi R Jackson ${ }^{11}$, Alisha Wilkens ${ }^{11}$, Ghayda Mirzaa ${ }^{10}$, Noriko Salamon $^{12}$, Jeff Abramson ${ }^{13,14}$, Elaine H Zackai ${ }^{11}$, Ian Krantz ${ }^{11}$, A Micheil Innes ${ }^{3}$, Stanley F Nelson ${ }^{1,2,4,6}$, Wayne W Grody ${ }^{1,2,4,6}$ and Fabiola Quintero-Rivera ${ }^{\star, 1,2}$

Dual-specificity tyrosine-(Y)-phosphorylation-regulated kinase $1 \mathrm{~A}(D Y R K 1 A)$ is a highly conserved gene located in the Down syndrome critical region. It has an important role in early development and regulation of neuronal proliferation. Microdeletions of chromosome 21q22.12q22.3 that include DYRK1A (21q22.13) are rare and only a few pathogenic single-nucleotide variants (SNVs) in the DYRK1A gene have been described, so as of yet, the landscape of DYRK1A disruptions and their associated phenotype has not been fully explored. We have identified 14 individuals with de novo heterozygous variants of DYRK1A; five with microdeletions, three with small insertions or deletions (INDELs) and six with deleterious SNVs. The analysis of our cohort and comparison with published cases reveals that phenotypes are consistent among individuals with the 21q22.12q22.3 microdeletion and those with translocation, SNVs, or INDELs within DYRK1A. All individuals shared congenital microcephaly at birth, intellectual disability, developmental delay, severe speech impairment, short stature, and distinct facial features. The severity of the microcephaly varied from -2 SD to -5 SD. Seizures, structural brain abnormalities, eye defects, ataxia/broadbased gait, intrauterine growth restriction, minor skeletal abnormalities, and feeding difficulties were present in two-thirds of all affected individuals. Our study demonstrates that haploinsufficiency of DYRK1A results in a new recognizable syndrome, which should be considered in individuals with Angelman syndrome-like features and distinct facial features. Our report represents the largest cohort of individuals with DYRK1A disruptions to date, and is the first attempt to define consistent genotype-phenotype correlations among subjects with $21 \mathrm{q} 22.13$ microdeletions and DYRK1A SNVs or small INDELs.

European Journal of Human Genetics (2015) 23, 1473-1481; doi:10.1038/ejhg.2015.71; published online 6 May 2015

\section{INTRODUCTION}

Protein kinases are key enzymes for the regulation of basic cellular processes in all eukaryotes. ${ }^{1}$ Dual-specificity tyrosine phosphorylationregulated kinase 1A (DYRK1A) (MIM 600855) is a protein kinase located in the Down syndrome critical region (DSCR) of chromosome 21 , and is a member of the highly conserved DYRK family of kinases. $^{1,2}$ DYRK proteins are dual-specificity kinases, which catalyze the phosphorylation of serine and threonine residues on exogenous substrates, as well as the phosphorylation of tyrosine residues in their own activation loop. DYRK1A has been extensively studied in both mouse (Dyrkla) and Drosophila (Mnb) orthologs. It is essential for neurogenesis, neuronal differentiation and proliferation, cell cycle, and synaptic plasticity. ${ }^{3}$ The extra copy of DYRK1A in individuals with Down syndrome (DS) accounts for the majority of their phenotypic features, ${ }^{4}$ and DYRK1A overexpression may be the underlying mechanism for abnormal brain development in DS individuals. ${ }^{5,6}$ Furthermore, data from both animal models and humans have suggested that decreased DYRK1A expression is also pathogenic. ${ }^{7-9}$ Pathogenic variants in DYRK1A resulting in haploinsufficiency of the gene have recently been proposed to cause intellectual disability (ID), mental retardation autosomal dominant type 7 (MIM 614104) in individuals with translocations, ${ }^{10}$ single-nucleotide

\footnotetext{
${ }^{1}$ Department of Pathology and Laboratory Medicine, David Geffen School of Medicine at University of California Los Angeles, CA, USA; ${ }^{2}$ UCLA Clinical Genomics Center, Los Angeles, CA, USA; ${ }^{3}$ Department of Medical Genetics, Cumming School of Medicine, University of Calgary, and Alberta Children's Hospital Research Institute for Child and Maternal Health, Calgary, AB, Canada; ${ }^{4}$ Department of Pediatrics, David Geffen School of Medicine at University of California Los Angeles, CA, USA; ${ }^{5}$ Kaiser Permanente, Fresno, CA, USA; ${ }^{6}$ Department of Human Genetics, David Geffen School of Medicine at University of California Los Angeles, CA, USA; ${ }^{7}$ Department of Pediatrics, Stanford University School of Medicine, Stanford, CA, USA; ${ }^{8} \mathrm{CHU}$ Nantes, Service de Génétique Médicale, and Inserm UMR957, Faculté de Médecine, Nantes, France; ${ }^{9}$ Institute of Mother and Child, Warsaw, Poland; ${ }^{10}$ Seattle Children's Research Institute, Seattle, WA, USA; ${ }^{11}$ Division of Human Genetics, Children's Hospital of Philadelphia, Philadelphia, PA, USA; ${ }^{12}$ Department of Radiology, David Geffen School of Medicine at University of California Los Angeles, CA, USA; ${ }^{13}$ Department of Physiology, David Geffen School of Medicine at University of California, Los Angeles, CA, USA; ${ }^{14}$ The Institute for Stem Cell Biology and Regenerative Medicine (inStem), National Centre for Biological Sciences-Tata Institute of Fundamental Research, Bangalore, Karnataka, India

*Correspondence: Dr F Quintero-Rivera, Department of Pathology and Laboratory Medicine, David Geffen School of Medicine at UCLA, 1000 Veteran Ave, Los Angeles, 90024 , CA, USA. Tel: +1 310794 9783; Fax: +1 310794 5099; E-mail: fquintero@mednet.ucla.edu

Received 12 September 2014; revised 5 March 2015; accepted 10 March 2015; published online 6 May 2015
} 
variants $(\mathrm{SNVs}),{ }^{11,12}$ and intragenic microdeletions ${ }^{11,13-17}$ disrupting only DYRK1A.

We describe the genotype-phenotype characterization of a new cohort of 14 individuals with de novo protein-damaging SNVs, small insertions or deletions (INDELs), or microdeletions involving DYRK1A. We demonstrate the pathogenic nature of its disruption and the delineation of this new recognizable syndrome.

\section{PATIENTS AND METHODS}

\section{Phenotypic analysis}

All samples and phenotype information were collected from individuals with various ethnic backgrounds (P1-P14, Table 1) after informed consent was obtained in accordance with each participating institutions' ethical committee protocols, and with a UCLA Institutional Review Board-approved protocol. Parental releases were obtained for the use of clinical information in this manuscript. A clinical geneticist provided phenotypic information for all patients. The clinical features of our cohort $(n=14$, Table 1$)$ are summarized and were assessed for both overlap and divergence among the cohort and compared with the 13 individuals in published reports, ${ }^{10-17}$ as shown in Supplementary Table S1.

\section{Genetic studies}

Clinical testing of peripheral blood samples from these individuals and their parents (as a trio) was performed using either clinical exome sequencing (CES) or chromosomal microarray analysis (CMA). Trio CES on P1 and P7 was performed at the UCLA Clinical Genomics Center (UCLA-CGC) using clinically validated protocols, as recently described. ${ }^{18}$ Variants of interest were selected on the basis of effect on protein, rarity, amino-acid (AA) conservation, and evaluation by PolyPhen2 and SIFT. For other patients (P2-P6, P8, P9) CES was performed on the proband only at different clinical laboratories (Supplementary Table S2). Clinically significant variants were confirmed using Sanger sequencing of the proband and both parents (Supplementary Figure S3), except for P6 whose father was unavailable for analysis. None of the nine DYRK1A variants were observed in 1633 exomes (761 families) in UCLA-CGC CES database or in publicly available NHLBI Exome Sequencing Project Exome Variant Server of 6500 exomes. CMA was performed on the five remaining individuals (P10-P14) using different standardized platforms (Supplementary Table S2). All deletions were confirmed on metaphase FISH (Supplementary Figure S1).

P12 and P14 subjects correspond to DECIPHER entry 257428 and 252 136, respectively. The data (genotype and phenotype) for P1-P11 and P13, corresponding to ClinVar entries: SCV000206782, SCV000206783, SCV000206784, SCV000206785, SCV000206786, SCV000206787, SCV000206788, SCV000206789, SCV000206790, SCV000206791, SCV000206792, SCV000206793 were submitted to ClinVar on 4 December 2014 (http://www.ncbi.nlm.nih. gov/clinvar/).

\section{Structural analysis}

The previously reported crystal structures of DYRK1A in complex with inhibitors harmine (PDB ID: 3ANR), INDY (PDB ID: 3ANQ), and a modified Pyrido-[2,3-d]-pyrimidine (PDB ID: 4QM1) were aligned and viewed using the program Coot. ${ }^{19}$ In silico modeling of DYRK1A SNVs was performed on PDB ID: 4QM1 where Leu at position 245 was replaced with $\operatorname{Arg}$ (p.(Leu245Arg)) and Leu at position 295 was replaced with Phe (p.(Leu295Phe)) utilizing standard fitting procedures in Coot. The modified models were analyzed by the MolProbity server, ${ }^{20}$ resulting in significant increase in residue clash score (4QM1 (wild type): 0.87, Leu295Phe: 1.9, and Leu245Arg: 2.77).

\section{RESULTS}

We observed that phenotypes were strikingly similar between individuals with a 21q22.11q22.3 microdeletion and those with SNVs involving only DYRK1A (Refseq NM_001396.3). Individuals shared borderline congenital microcephaly $(-2 \mathrm{SD}, 13 / 14)$, short stature (-1 to $-2.5 \mathrm{SD}, 13 / 14)$, global developmental delay (DD) (14/14), severe to moderate ID (13/14; the youngest patient (P9) shows no sign of ID yet at 21 months), severe speech impairment (speech was absent in $9 / 14$, and limited to few words in the remaining six individuals) and distinct facial features (Figure 1). Feeding difficulties (14/14), brain abnormalities (11/11), IUGR (11/14), minor skeletal issues (11/14), seizures (9/14), eye defects (9/14), and ataxia/broad-based gait (8/12) were present in most individuals. The severity of microcephaly varied from -1 to -4 SD at birth, and abnormally slow head growth caused the deviation from average to further increase over time ( -2 to $-5 \mathrm{SD}$ at the last follow-up). Seizures (9/14) were initially febrile, with ages of onset of 6-18 months, and then became generalized tonic-clonic. Feeding issues occurred with or without gastrointestinal (GI) defects, including constipation (9/14), gastroesophageal reflux disease (4/14), pyloric stenosis (2/14). Minor skeletal abnormalities include long tapered fingers (6/11), toe abnormalities (4/11), and scoliosis $(2 / 11)$. Eye defects ranged from esotropia, hypermetrotopia, myopia, strabismus, enophthalmia to more severe defects such as bilateral cataracts.

Brain MRI was performed on 11 individuals. The most common abnormalities found were small brain stem (9/11), enlarged ventricles (7/11), microcephaly (3/11), hypoplastic pituitary stalk $(6 / 11)$, white matter hypomyelination (5/11), hypoplastic corpus callosum (3/11), cortical atrophy/ frontal lobe atrophy (3/11), gliosis (3/11), and thin optic chiasm (2/11) (Figure 2). These findings are indicative of global cerebral underdevelopment or hypomyelination.

Some individuals presented with less common features including susceptibility to infections by non-common pathogens (6/14), endocrine problems (hypothyroidism, premature thelarche, and low growth hormone levels, 4/14), anxiety (4/14), hand stereotypies (4/14), aggressive behavior (3/14), happy demeanor (2/14), and autism spectrum disorder $(2 / 14)$.

The distinct facial features included sparse scalp hair, bitemporal narrowing, deeply set eyes, peri-orbital fullness, short nose with high nasal root and pointed nasal tip, prominent ears with underdeveloped ear lobes, variations of philtrum (short, prominent or tented), thin vermillion border of upper lip (11/14), short chin with horizontal crease and/or chin dimple (Figure 1). In P6, microscopic hair examination showed hypopigmentation.

There were some strikingly similar features between members of our cohort and the features of previously reported individuals with a similar genotype, ${ }^{10-17}$ indicating that this is a recognizable syndrome. Microcephaly, IUGR, brain abnormalities consistent with cerebral hypomyelination, global DD, ID, severe speech delay, seizures, broadbased gait, short stature, minor skeletal anomalies, and distinct facial gestalt were the most commonly shared features.

All patients in our cohort had an initial extensive genetic workup that was inconclusive. This is well illustrated by the differential diagnoses and the genetic-testing referrals for one or more of Angelman syndrome (AS), fragile $\mathrm{X}$, Mowat-Wilson and $\mathrm{AD}$ hyperIgE recurrent infection syndrome, screening for metabolic disorders, single gene sequencing, and chromosomal breakage studies, among others, all of which were negative or unremarkable (Supplementary Table S2). The most common differential diagnosis was AS. However, unlike individuals in our cohort, in AS patients, the prenatal history, fetal development, birth weight, and occipital frontal circumference (OFC) at birth are usually normal, and postnatal microcephaly is absolute in half by 1 year or relative by 2 years of age.

All individuals, seven males and seven females between age 17 months and 18.5 years, had either deletion of or proteindamaging variants in DYRK1A. We identified five microdeletions ranging from $1.53 \mathrm{Mb}$ to $4.17 \mathrm{Mb}$ in size (P10-P14), three missense variants (P7-P9), three nonsense variants (P1-P3), two frameshift 


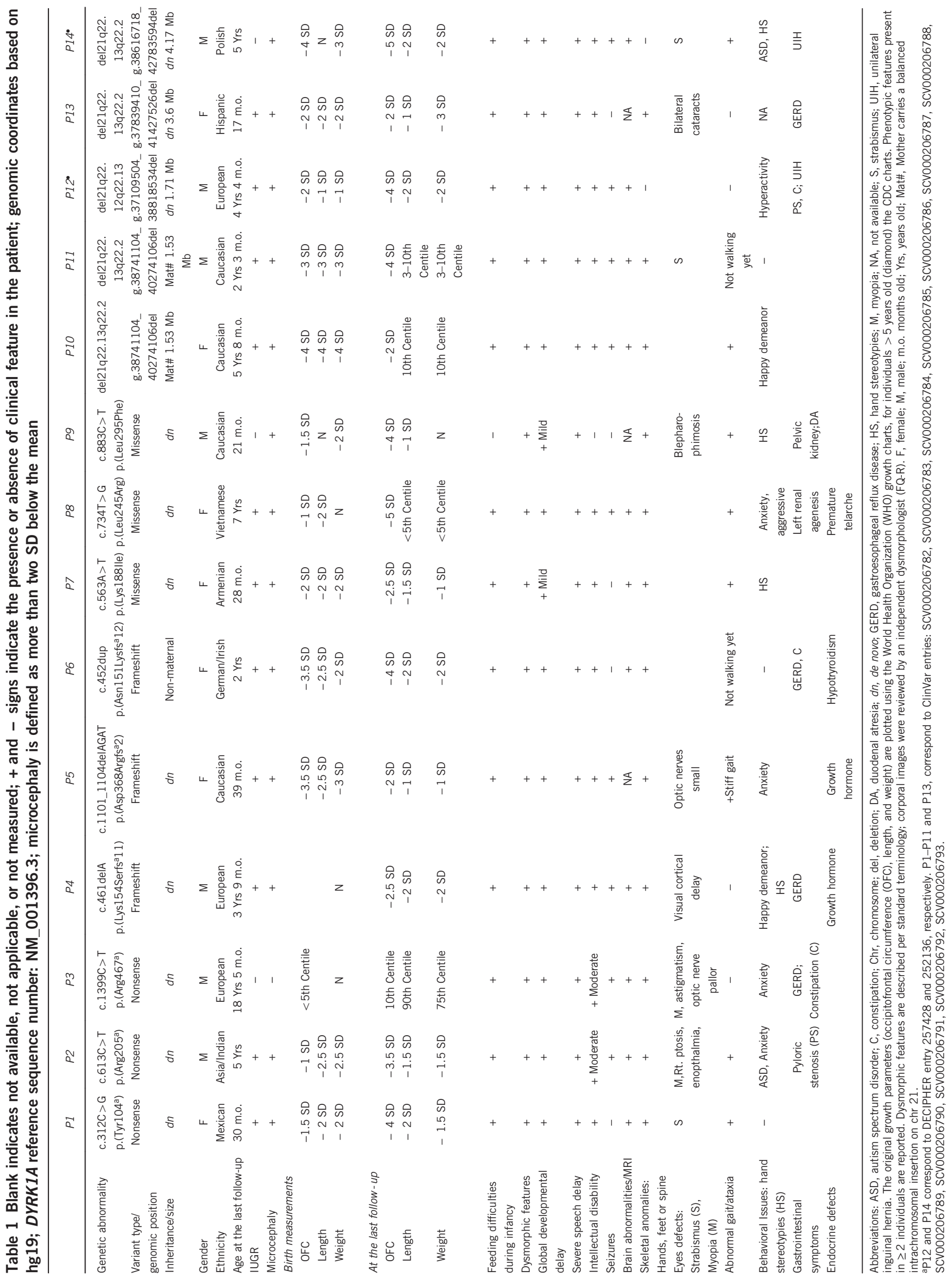



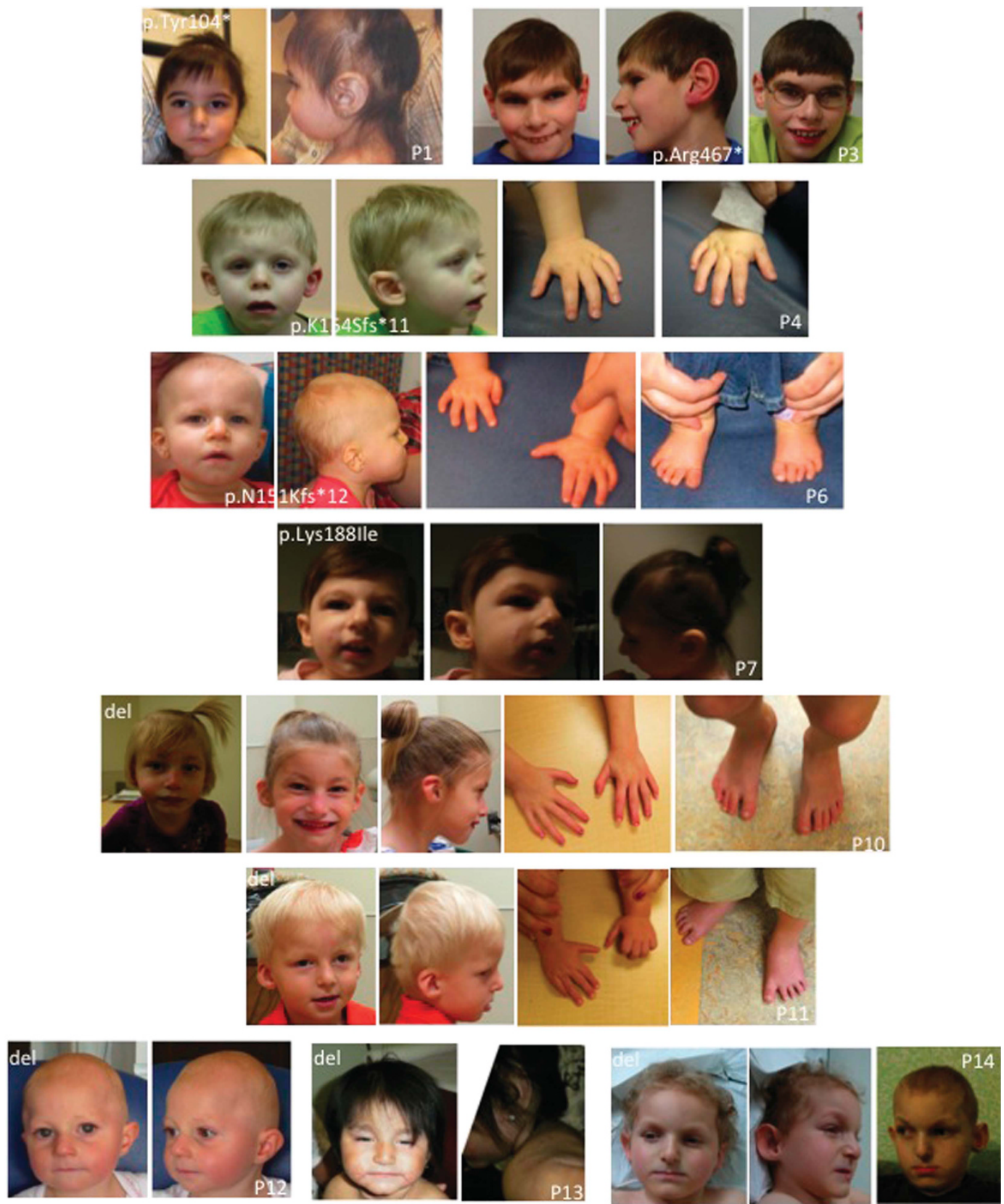

Figure 1 Facial features, hand and feet abnormalities of individuals with DYRK1A SNVs, INDELs, and microdeletions. Note the sparse scalp hair, bitemporal narrowing, deeply set eyes, peri-orbital fullness, prominent nasal bridge and pointed nasal tip, prominent ears with underdeveloped ear lobes, variations of philtrum (short in P3 and P10, prominent and tented in P4, tented in P7), thin vermillion border of the upper lip, short chin with horizontal crease and/or chin dimple. P4 has slightly tapered fingers (STF), mild fetal finger pads (not shown), broad fingertips; P6 has STF, mild clinodactyly bilaterally (MCB), curved 4th toes on the left (CTL); P10, at ages 3 y.o. and 8.7 y.o., has STF, MCB, CTL; P11 has STF and small feet. P3 at ages 9.9 y.o., and 12 y.0.; P14 at ages 5 y.o. and 11 y.o. Photographs of P2, P5, P8, and P9 were not available for publication.

deletions (P4 and P5), and one frameshift duplication (P6) where the nonsense and frameshift variants result in premature truncation of the protein. All genetic alterations were de novo and heterozygous, and are described in detail in Table 1 and Figure 3. Three missense variants (P7-P9) occurred within the $5^{\prime}$-end of the gene coding for the protein kinase domain. In silico analysis of human DYRK1A crystal structures shows that these AA substitutions (p.(Lys188Ile), p.(Leu245Arg), p.(Leu295Phe)) reside in or in close proximity to the ATP-binding site (Figure 4). p.Lys188 is located on the N-terminal lobe where it coordinates Glu203 and orients the $\alpha$ - and $\beta$-phosphate groups of ATP. ${ }^{21}$ Moreover, p.Lys188 is an essential residue for kinase activity as demonstrated by p.(Lys188Arg) variant that abolishes kinase function. ${ }^{22,23}$ To further assess the influence of these SNVs, a structural alignment of DYRK1A in complex with three different inhibitors (harmine, PDB ID: 3ANR; INDY, PDB ID: 3ANQ; and a modified Pyrido-[2,3-d]-pyrimidine, PDB ID: 4QM1) was performed in the program $\operatorname{Coot}^{19}$ using standard structural alignment protocols. In these structures, p.Lys 188 directly coordinates the pyridine nitrogen 

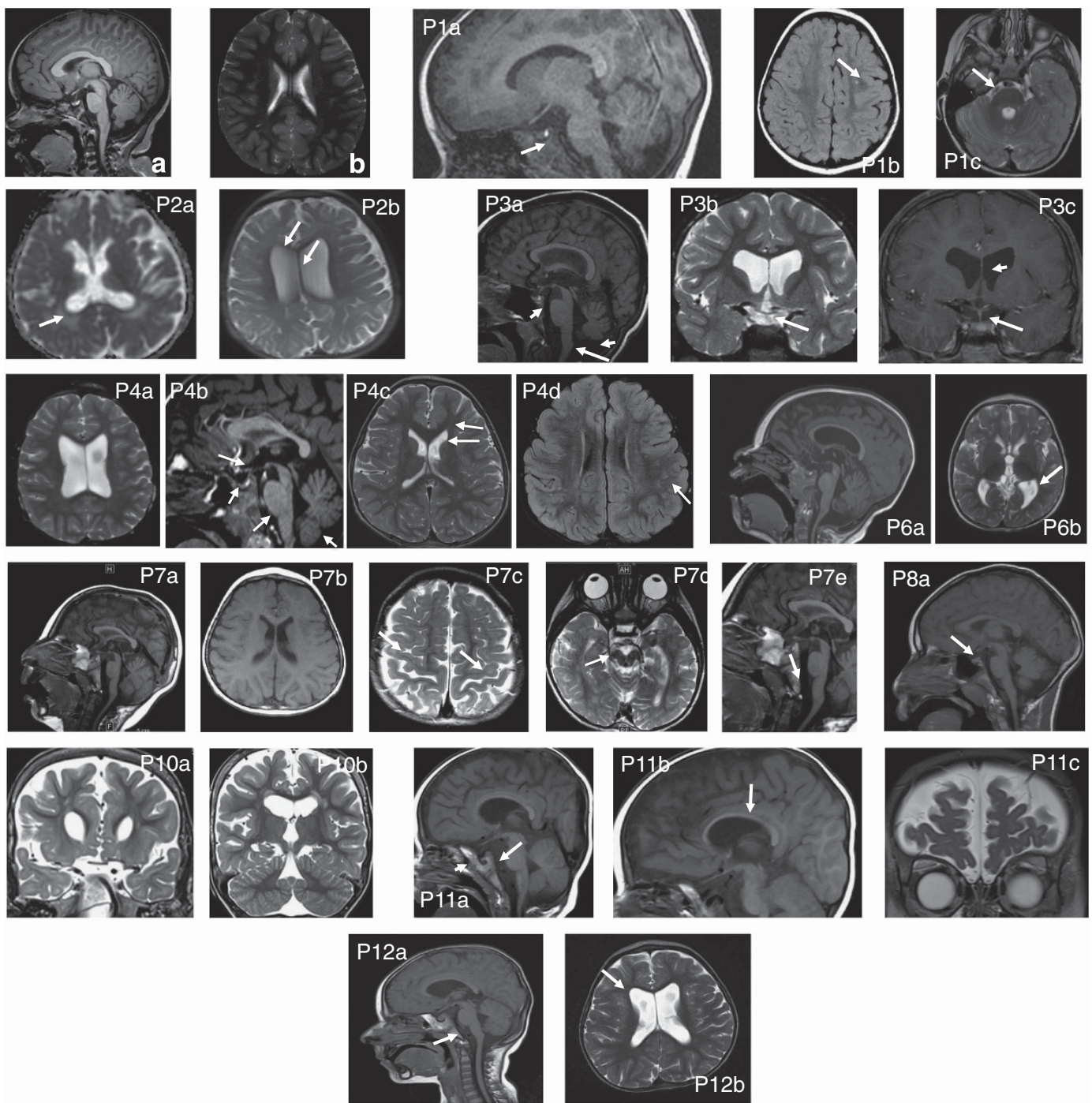

Figure 2 Brain magnetic resonance imaging (MRI) findings - T1 and T2 images. (a, b): normal brain architecture of a 5-year old. MRIs obtained at the following ages: P1 36 m.o.; P2 18 m.o.; P3 10 y.o.; P4 4.2 y.o., P6 17 m.o.; P7 21 m.o.; P8 6 y.o.; P10 35 m.o.; P11 16 m.0.; P12 25 m.o. Pla - hypoplastic pituitary gland (HPG), P1b - gliosis, P1c - small brain stem (SBS) indicated by prominent prepontine cistern; P2a - large ventricles (LV), white matter hypomyelination (WMH), P2b - hypoplastic (thin) corpus callosum (HCC); P3a - SBS, thinning upper cervical cord, P3b - thin optic chiasm (TOC), P3c - LV, HPG; P4a - LV, P4b - TOC, HPG, SBS, P4c - frontal lobe atrophy (FLA), prominent frontal horns, P4d-WMH, gliosis; P6a - HCC, SBS, P6b - LV, WMH; P7a - microcephaly (MC), P7b - mild LV, P7c - WMH, P7d - SBS, P7e - SBS; P8a - MC, HPG; P10ab - LV, SBS, brain atrophy and WMH, gliosis in periventricular regions (not shown); P11a - HPG (arrowhead), SBS (white arrow), P11b - HCC, P11c - FLA; P12a - MC, SBS, HPG; P12b LV, FLA. Original MRIs were reviewed by a UCLA Pediatric Neuroradiologist (NS).

of harmine, the hydroxyl group of INDY, and the ester carbonyl oxygen of the modified Pyrido-[2,3-d]-pyrimidine compound and all complexes reside in a similar location. By all structural and functional evidence, a change of p.Lys188 to Ile would prevent ATP-dependent phosphorylation.

The other missense variants, p.(Leu245Arg) and p.(Leu295Phe), are located in the C-terminal lobe on a three-stranded $\beta$-sheet (242-245, 293-296, and 303-306), which contours the ATP-binding site (Figure 4). It is noteworthy that p.Leu245 is positioned at the $\mathrm{C}$-terminal portion of the hinge region, which connects the $\mathrm{N}$ - and C-terminal lobes. Substitution of a Leu for the bulkier side-chains of Arg and Phe generates steric clashes that may disrupt the integrity of the active site and prevent ATP binding. This hypothesis is supported by in silico modeling of p.(Leu245Arg) and p.(Leu295Phe) SNVs into crystal structure, 4QM1. Analysis using the MolProbity server ${ }^{20}$ revealed a substantial increase in the overall clash score (4QM1 (wild type): 0.87, Leu295Phe: 1.9, and Leu245Arg: 2.77). On closer inspections, the p.(Leu295Phe) variant causes a serious steric overlap in excess of $1.5 \AA$ with p.Leu248, whereas the p.(Leu245Arg) variant causes a similar steric overlap with p.Ile303 (1.5 $\AA$ ) and p.Ile293 (1.2 $\AA$ ). In total, each of the three missense variants (P7-P9) could abolish catalytic activity of DYRK1A by disrupting its ability to bind ATP. The fact that the $5^{\prime}$-end of the protein kinase domain is otherwise depleted of similar missense variants in the population, as shown in Figure 3, suggests that the mutational burden is overall higher in this region. P3 has a nonsense variant leading to a premature stop of protein synthesis at p.Arg467 but growth parameters (OFC and length) are not as decreased like in the other patients. p.Arg467 is positioned at the far $\mathrm{C}$ terminus of the protein kinase domain, which is not in the proximity of the active site. However, this p.Arg467 forms 


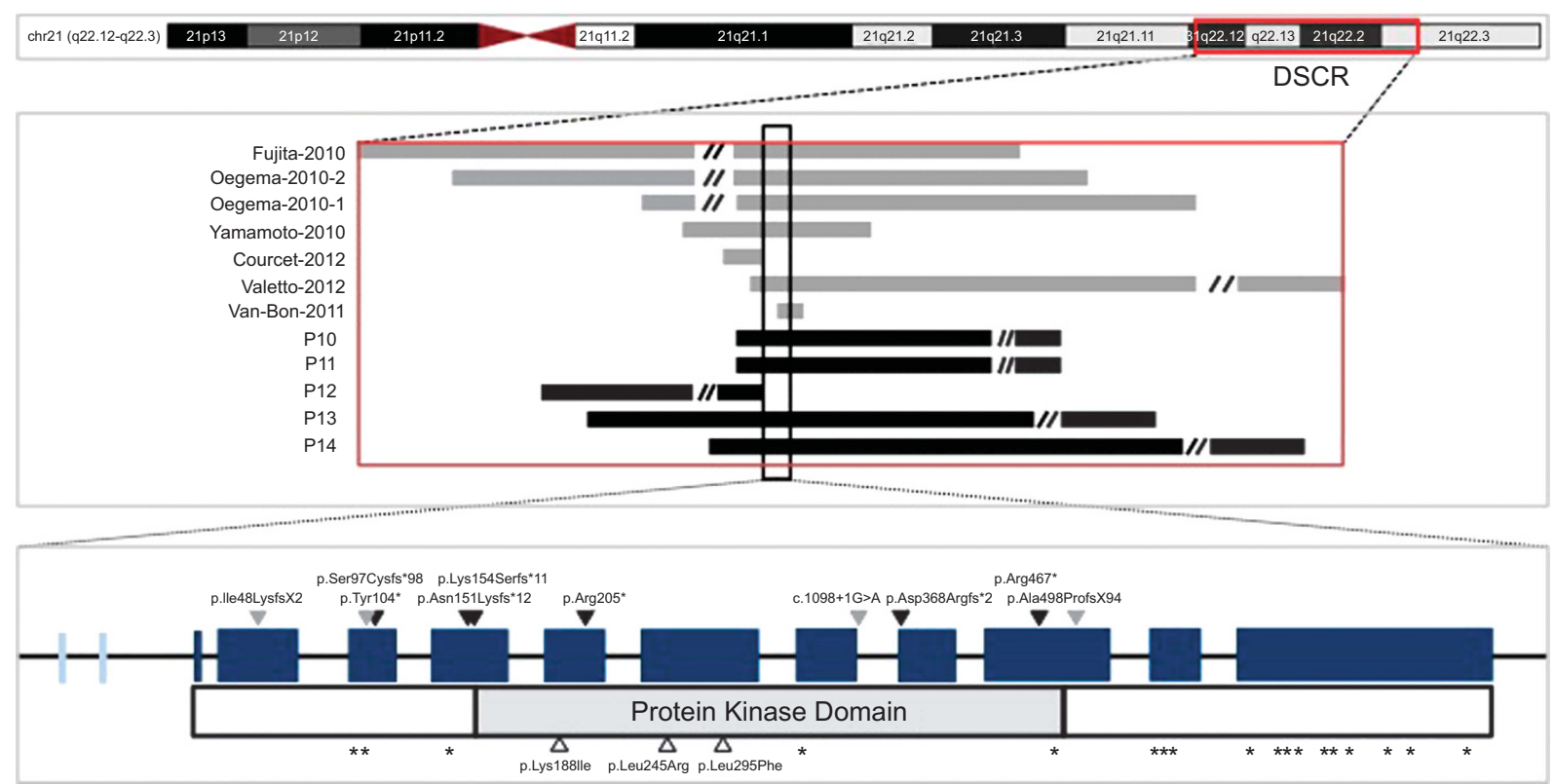

Figure 3 Schematic representation of all genetic alterations in this report and those previously reported in the literature. Upper panel: chromosome 21 cytoband (hg19); boxed area represents DSCR. Middle panel: 21q22.13 deletion in different patients (un-scaled illustration); gray: published microdeletions, as indicated by the author and year; black: five microdeletions including DYRK1A. The black box across the deletions shows the position of DYRK1A gene. Lower panel: DYRK1A single-nucleotide variants and small INDELs with the scheme of corresponding amino-acid changes; blue box: the 11 exons of the DYRK1A gene. The width of the box represents the number of nuclear acids; light blue box: two alternative splicing sites; black solid arrowheads: Nonsense or frameshift variants identified in our patients; gray solid arrowheads: nonsense or frameshift variants identified in cases from published literature; white arrowheads: missense variants identified in our patients; (*)asterisks: missense variant positions in normal individuals in EVS that are predicted to be damaging by PolyPhen2.

a salt bridge with p.Glu331, which is near to the autophosphorylated Tyr 321, and may interfere with the activation of the kinase (data no shown). Additional molecular functional studies to determine the kinase activity and the phosphorylation pattern in the cells with engineered missense variants should probe these predictions.

Microdeletions disrupting DYRK1A were discovered in five individuals (P10-P14); all microdeletions contain most of the DSCR, which spans about $7 \mathrm{Mb}$ of chromosome 21 (position 35.8 to $42.3 \mathrm{Mb}$, hg19), and includes all genes between RCNA1 and MX1. ${ }^{24}$ In all but one individual, the microdeletion encompassed the entire DYRK1A gene. In P12, the deletion only removes the first exon and part of the first intron of DYRK1A (NG_009366.1). Of note, the mother of P10 and P11 has a rare intrachromosomal insertion of part of the DSCR into the short arm of chromosome 21, and both siblings inherited the unbalanced maternal recombinant that resulted in the loss of the inserted fragment, and therefore, deletion of 21q22.13q22.2. Interestingly, the sister (P10) is mosaic for the deletion with a normal cell line in the minority, and has a dizygotic unaffected twin brother (Supplementary Figure S1). A mosaic deletion was also detected in $\mathrm{P} 13$ by FISH studies, and in two published cases ${ }^{14,16}$ (Supplementary Figure S2). P13 and P14 have the largest deletions. We screened the other genes deleted in these individuals for an OMIM phenotype, and found two with disease causation and one with an association. Homozygous SNVs in CLDN14/DFNB29 are associated with deafness, autosomal recessive (AR) 29 (MIM 614035), and in HLCS with AR holocarboxylase synthetase deficiency (MIM 253270). None of these genes explains the phenotypes we are describing in this cohort.

For comparison, an additional seven individuals have been previously reported with microdeletions encompassing DYRK1A, ranging from $52 \mathrm{~Kb}$ to $4.9 \mathrm{Mb}$ in size, and four with truncating SNVs. ${ }^{10-16}$ These are included in Supplementary Table S1. Specific evidence supporting the role of DYRK1A individuals includes the deletion of the non-coding 5'-UTR (NM_130436.3) in one published case, ${ }^{11}$ as well as the disruption of DYRK1A by translocation in two cases reported by Moller et al. ${ }^{10}$ Furthermore, in the Database of Genomic Variants, there are only two self-reported phenotypically normal individuals with exonic losses of DYRK1A ( $8 \mathrm{~Kb}$ and $181 \mathrm{~Kb})$. There are no segmental duplications flanking the deletion breakpoints.

With one exception, we did not find other molecular events that could potentially explain the condition in these 14 individuals. The exception is P8 (with a nonsense SNV in DYRK1A) who may have a di-genic phenotype; in addition to the DYRK1A-related phenotype, she has left renal agenesis and a high palate, and her mother has bilateral pre-auricular pits. A missense variant of uncertain clinical significance in SIX5 (MIM 600963), maternally inherited and known to cause Brachiotorenal syndrome 2 (MIM 610896) was detected by CES, and possibly contributes to this patient's phenotype. Other SNVs or CNVs inherited from one unaffected parent and likely benign were reported in subjects P2-P6 and P9 (Supplementary Table S2).

In summary, although we cannot exclude the potential contribution of other deleted genes to patients' phenotype, we suggest that the haploinsufficiency of DYRK1A and loss of function variants is sufficient for the clinical manifestation of most of the neurodevelopmental abnormalities presented above.

\section{DISCUSSION}

DYRK1A has been studied extensively in the last decade because of its association with neuronal deficits, dendritic atrophy, spinal dysgenesis, precocious Alzheimer-like neurodegeneration, and cognitive deficits in DS individuals. The gene has 13 exons and spans $\sim 147.8 \mathrm{~Kb}$ of genomic DNA. Alternative splicing generates several transcript variants, differing in either $5^{\prime}$ UTR or $3^{\prime}$ coding region and encoding at 
a

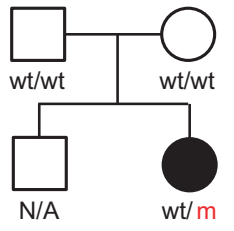

P7

b

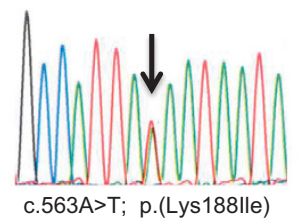

C

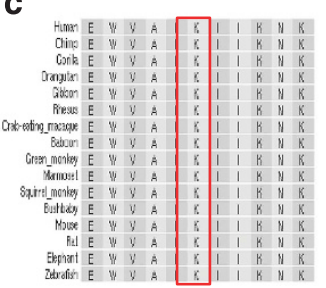

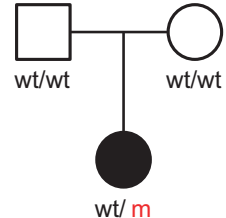

P8

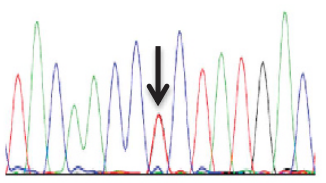

c.734T>G; p.(Leu245Arg)

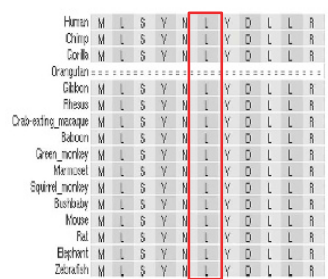

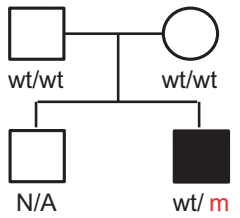

P9
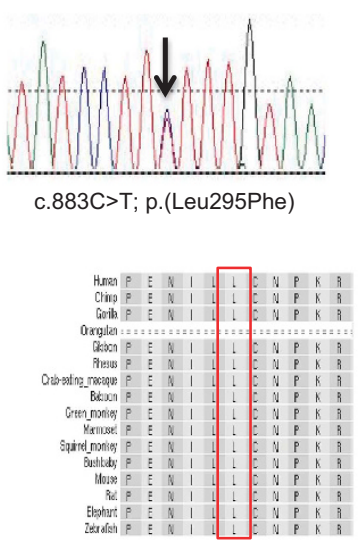

d

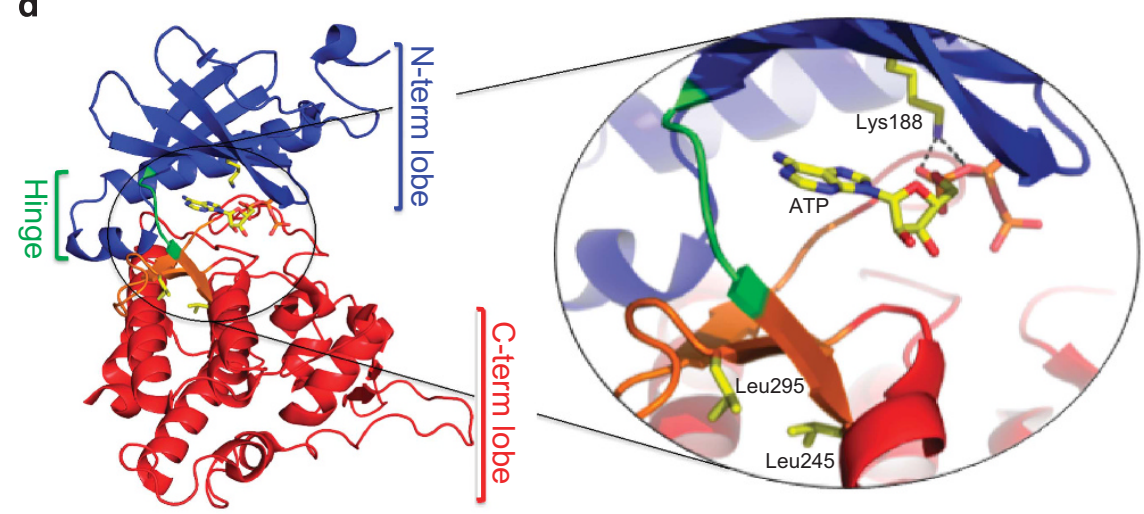

e

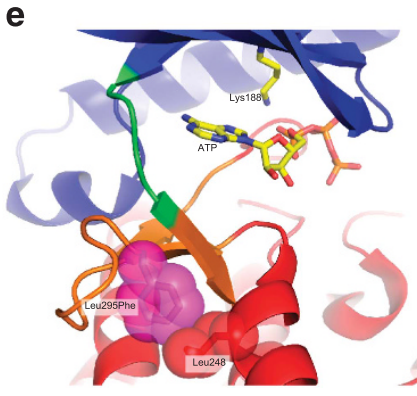

f

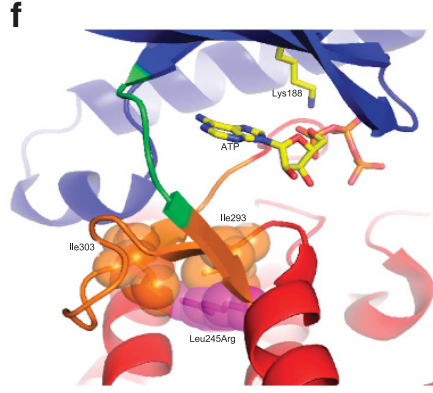

Figure 4 Features of the three missense variants identified in DYRK1A. (a) The pedigree of three families with missense variants. (b) The Sanger sequencing traces of two of these variants. (c) Amino-acid alignments at the variant position (red box) and surrounding bases across 16 species as indicated on the left. (d) Overview of the human DYRK1A protein structure (PDB ID: 4MQ1). The active site (inset) is sandwiched between the N-terminal (blue) and C-terminal lobes (red), which are connected by a short hinge segment (green). The three residues mutated in patients (Lys188, Leu245, and Leu295) and the ATP (modeled from PDB ID: 1ATP) are shown as ball-and-stick configuration colored by atom. Lys188 coordinates (black dashed lines) the $\alpha$ - and $\beta$-phosphates of ATP. (e, f) Steric clashes caused by variants of Leu295Phe and Leu245Arg in relation to the active site. (e) Leu295Phe variant (magenta) causes a clash with Leu248 (red). (f) Leu245Arg (magenta) causes a clash with Ile303 (orange) and Ile293 (orange).

least five different isoforms. The canonical transcript of DYRK1A encodes a nuclear protein of $763 \mathrm{AA}$. It contains a protein kinase domain from AA 159 to AA 479. DYRK1As' catalytic activity is not modulated by phosphorylation, in contrast to most kinases whose activity is turned on or off by kinase or phosphatase. ${ }^{6}$ However, to achieve full kinase activity, one fully conserved tyrosine (Tyr 321) in the activation loop must be constitutively autophosphorylated immediately after translation. ${ }^{25}$ At least 20 DYRK1A exogenous substrates and/or interacting proteins have been identified, including Caspase 9, cyclin D1, c-AMP response element binding protein (CREB), CRY2, 
E2F1, FOXO1, GLI1, GSK3, INI1/SNF5, NFAT, RAS, RAF, MEK1, SRSF6, STAT3, SJI1, and SIRT1. ${ }^{5}$ The overexpression of DYRK1A affects the expression of 239 genes through the NRSF/REST-SWI/SNF chromatin-remodeling complex, a key regulator of pluripotency and neuronal differentiation, indicating an essential role of this kinase in cell cycle and in brain development. ${ }^{26}$ Like many kinases, DYRK1A is involved in the control of many critical signaling pathways of cell proliferation, such as AKT, mitogen-activated protein kinase, MAPK/ ERK, ${ }^{27}$ and STAT3, all of which are related to neuron differentiation and development. ${ }^{28,29}$ It enhances the MAPK cascade by forming a complex with Ras, B-Raf and MEK $1 .{ }^{30}$ It also interacts with GLI1 (MIM 165220), which mediates SHH signaling and may have a role in craniofacial development and digital development, as well as development of the central nervous system and GI tract. Furthermore, the regulation of NFATc1 by DYRK1A alone is sufficient to control bone homeostasis and skeletal development. ${ }^{31}$ No relevant functional redundancy of the different DYRK members has been reported. ${ }^{1,32}$

The Dyrkla $( \pm)$ mouse is a phenocopy of the human phenotype. The pyramidal cells of the cerebral cortex are considerably smaller, less branched and less spinous in the cortex of Dyrk $1 A \pm$ mice than in normal mice, causing the neurons to receive fewer excitatory GABA and glutamatergic inputs. ${ }^{9}$ Moreover, DYRK1A modulates the activity of the CREB. ${ }^{8,9}$ CREB phosphorylation is increased in rodent epilepsy models, and in the seizure onset regions of humans with epilepsy. ${ }^{33}$ Dyrk $1 a \pm$ mice also have decreased in the size of the somatosensory cortex and thalamus, increase in the astroglial population (as a result of crowding of the neuronal cells), increased anxiety, changes in emotional behavior, and delayed reflexes. In the $m n b$ fly, also phenotypically similar to the human phenotype, efficient synaptic vesicle recycling is promoted. ${ }^{7,9,34}$ Retino-cortical visual processing defects and body and wing size reduction are seen in both Dyrkla $( \pm)$ and the mnb fly. ${ }^{35,36}$ Dyrkla-haploinsufficient mice show severe glucose intolerance and decreased beta cell proliferation. ${ }^{37}$ Finally, in the Dyrkla mouse and the $m b n$ fly AKT-mediated insulin signaling pathway suppresses FOXO-mediated neuropeptide $\mathrm{Y}$ expression, which results in decreasing food intake. ${ }^{38}$

We propose that the IUGR, postnatal growth retardation, microcephaly, seizures, behavior abnormalities, ataxia, eye defects, brain findings (gliosis, cortex atrophy, and white matter hypomyelination), minor skeletal anomalies, feeding, and GI issues are consistent with the gene dysregulation of multiple DYRK1A interactors, as these findings are all present in both Dykrla $( \pm)$ mice and our cohort.

The five microdeletions described in this cohort involving DYRK1A are telomeric and distinct from the 21q22.11q22.12 microdeletion (a.k.a. Braddock-Carey syndrome (BCS), (MIM 601399), spanning coordinates 32.5-37.5 (hg19)), which always includes RUNX1 (MIM 151385) (36.1-36.4, hg19). The BCS results in a different phenotype, which includes congenital thrombocytopenia, Pierre Robin sequence, agenesis of the corpus callosum, DD, and facial dysmorphisms. ${ }^{39}$ BCS is always non-mosaic. ${ }^{40}$ In our cohort and in the previously reported patients (with one exception ${ }^{15}$ ) RUNX1 is intact. The BCS microdeletion includes part of the centromeric end of the DSCR but does not include DYRK1A. We hypothesize that patients with microdeletions of 21q22.13 (position 37.5-42.7 Mb, hg19), which include that DYRK1A will exhibit similar phenotype to those in our cohort.

Courcet et al. estimated that among patients with microcephaly, seizures, and absent language, DYRK1A is mutated in 1:70 (1.4\%). The frequency of DYRK1A disruption in patients with clinical indication of DD, ID with or without dysmorphic features was 2 in 170 CES cases, ${ }^{41}$ and 1 in 719 patients tested by SNP-CMA in the UCLA-CGC.
Our study raises practical and important issues about the current clinical interpretation of variants of uncertain significance and coding region-focused analyses of SNP-CMA and CES results. Two of the published cases, ${ }^{13,17}$ and a third one in DECIPHER (258106) have exonic pathogenic deletions $(52,69$, and $20 \mathrm{~Kb}$, respectively) confined to the $5^{\prime}$ or $3^{\prime}$ end of DYRK1A (exon 9-11, exon 1, exon 7-11, respectively), highlighting the limitation of routine CMA using stringent cutoffs ( $>50$ to $>400 \mathrm{~Kb}$ ). Although, this $\mathrm{CNV}$ was not detected in the largest prenatal CMA study by Wapner et al., ${ }^{42}$ the routine use of prenatal CMA in fetuses with IUGR, polyhydramnios, microcephaly, and hypoplasia corpus callosum may detect more affected individuals.

We observed hypoplasia of pituitary stalk on MRI (Figure 2), and endocrine issues, and short stature (13/14), for which some patients are on growth hormone $(2 / 14)$ or on thyroid hormone (1/14) replacement. It is plausible that these issues are secondary to the dysfunction of hypothalamus-pituitary-hypophysis axis. Therefore, we suggest a baseline endocrine evaluation in patients with DYRK1A haploinsufficiency syndrome. In addition, we recommend monitoring of glucose levels, as Dyrkla ( \pm ) mice show a diabetic profile. ${ }^{37}$ Finally, there is a potential for a pre- and postnatal pharmacological treatment to improve these individuals. Excitation/inhibition balance and learning are modified by Dyrkla gene dosage. ${ }^{43} \mathrm{~A}$ recent study shows that a DYRK1A inhibitor rescues the cognitive deficit in DS mouse models and in humans. ${ }^{44} \mathrm{~A}$ similar approach enhancing DYRK1A expression may benefit individuals with this new syndrome. Furthermore, prenatal treatment of DS mice in utero has shown therapeutic effects that persisted to adulthood. ${ }^{45}$

In summary, we propose that the combination of clinical findings observed in 14 affected individuals from 13 independent families (summarized in Table 1, with additional clinical information in Supplementary Table S1 available online) define a distinctive new syndrome due to DYRK1A disruption and likely resulting in haploinsufficiency. Individuals with this syndrome display signs of IUGR, congenital microcephaly, typical gestalt, short stature, ID, severe speech delay, seizures, global DD, global brain underdevelopment/ hypomyelination, eye and skeletal defects, and feeding difficulties. The mouse and fly phenocopies support this hypothesis. DYRK1A disruption and genome-wide testing should be considered in individuals with Angelman-like features with congenital microcephaly and facial gestalt. Re-evaluation of patients with AS-like features and negative genetic testing should be considered, as this new DYRK1A haploinsufficiency syndrome might be more common than previously thought.

\section{CONFLICT OF INTEREST}

The authors declare no conflict of interest.

\section{ACKNOWLEDGEMENTS}

We would like to thank the families, the patients, and clinical staff at all locations for participation in this study. We are very grateful to Dr Xinmin Li, Jonathan David, Vanina Tomasina, Traci Toy, and Lynn Yang for their technical assistance on CMA, CES, and FISH analysis, and the UCLA-CGC Genomic Data Board members for their contribution to exome data interpretation. We thank Drs Aviv Paz and Thorsten Althoff for their assistance in structure analysis and figure generation, Dr Sulagna Saitta for useful discussion, and Dr Kevin M Squire for his critical reading of this manuscript. This work was partially presented at the 2014 American College of Medical Genetics and Genomics annual meeting, 26-29 March, Nashville, TN, USA, and the 35th David Smith Workshop on Morphogenesis and Dysmorphology at University of Madison, WI, 25-30 July, 2014. This study was supported by 
NIH grant GM078844 (JA), and the UCLA Department of Pathology translational research fund (FQ-R).

1 Aranda S, Laguna A, de la Luna S: DYRK family of protein kinases: evolutionary relationships, biochemical properties, and functional roles. FASEB J 2011; 25: 449-462.

2 Becker W, Weber Y, Wetzel K, Eirmbter K, Tejedor FJ, Joost HG: Sequence characteristics, subcellular localization, and substrate specificity of DYRK-related kinases, a novel family of dual specificity protein kinases. J Biol Chem 1998; 273: 25893-25902.

3 Song WJ, Sternberg LR, Kasten-Sportes C et al: Isolation of human and murine homologues of the Drosophila minibrain gene: human homologue maps to $21 \mathrm{q} 22.2$ in the Down syndrome 'critical region'. Genomics 1996; 38: 331-339.

4 Shapiro BL: The Down syndrome critical region. J Neural Transm Supp/ 1999; 57 41-60.

5 Tejedor FJ, Hammerle B: MNB/DYRK1A as a multiple regulator of neuronal development. FEBS J 2011; 278: 223-235.

6 Becker W, Sippl W: Activation, regulation, and inhibition of DYRK1A. FEBS J 2011; 278: 246-256.

7 Fotaki V, Dierssen M, Alcantara S et al: Dyrk1A haploinsufficiency affects viability and causes developmental delay and abnormal brain morphology in mice. Mol Cell Biol 2002; 22: 6636-6647.

8 Dierssen M, de Lagran MM: DYRK1A (dual-specificity tyrosine-phosphorylated and -regulated kinase $1 \mathrm{~A}$ ): a gene with dosage effect during development and neurogenesis. ScientificWorldJournal 2006; 6: 1911-1922.

9 Benavides-Piccione R, Dierssen M, Ballesteros-Yanez I et al: Alterations in the phenotype of neocortical pyramidal cells in the Dyrk1A+/- mouse. Neurobiol Dis 2005; 20: 115-122.

10 Moller RS, Kubart S, Hoeltzenbein M et al: Truncation of the Down syndrome candidate gene DYRK1A in two unrelated patients with microcephaly. Am J Hum Genet 2008; 82: 1165-1170

11 Courcet JB, Faivre L, Malzac P et al: The DYRK1A gene is a cause of syndromic intellectual disability with severe microcephaly and epilepsy. J Med Genet 2012; 49: 731-736.

12 O'Roak BJ, Vives L, Girirajan S et al: Sporadic autism exomes reveal a highly interconnected protein network of de novo mutations. Nature 2012; 485: 246-250.

13 van Bon BW, Hoischen A, Hehir-Kwa J et al: Intragenic deletion in DYRK1A leads to mental retardation and primary microcephaly. Clin Genet 2011; 79: 296-299.

14 Yamamoto T, Shimojima K, Nishizawa T, Matsuo M, Ito M, Imai K: Clinical manifestations of the deletion of Down syndrome critical region including DYRK1A and KCNJ6. Am J Med Genet A 2011; 155A: 113-119.

15 Fujita $\mathrm{H}$, Torii $\mathrm{C}$, Kosaki $\mathrm{R}$ et al: Microdeletion of the Down syndrome critical region at 21q22. Am J Med Genet A 2010; 152A: 950-953.

16 Oegema R, de Klein A, Verkerk AJ et al: Distinctive phenotypic abnormalities associated with submicroscopic 21q22 deletion including DYRK1A. Mol Syndromol 2010; 1: 113-120.

17 Valetto A, Orsini A, Bertini V et al: Molecular cytogenetic characterization of an interstitial deletion of chromosome 21 (21q22.13q22.3) in a patient with dysmorphic features, intellectual disability and severe generalized epilepsy. Eur J Med Genet 2012; 55: 362-366

18 Strom SP, Gorin MB: Evaluation of autosomal dominant retinal dystrophy genes in an unaffected cohort suggests rare or private missense variants may often be benign. Molecular vision 2013; 19: 980-985.

19 Emsley P, Lohkamp B, Scott WG, Cowtan K: Features and development of Coot. Acta crystallogr Sect D Biol Crystallogr 2010; 66: 486-501.

20 Chen VB, Arendall WB3rd, Headd JJ et al: MolProbity: all-atom structure validation for macromolecular crystallography. Acta Crystallogr Sect D Biol Crystallogr 2010; 66: 12-21.

21 Endicott JA, Noble ME, Johnson LN: The structural basis for control of eukaryotic protein kinases. Annu Rev Biochem 2012; 81: 587-613.
22 Kentrup H, Becker W, Heukelbach J et al: Dyrk, a dual specificity protein kinase with unique structural features whose activity is dependent on tyrosine residues between subdomains VII and VIII. J Biol Chem 1996; 271: 3488-3495.

23 Wiechmann S, Czajkowska H, de Graaf K, Grotzinger J, Joost HG, Becker W: Unusual function of the activation loop in the protein kinase DYRK1A. Biochem Biophys Res Commun 2003; 302: 403-408.

24 Berto GE, lobbi C, Camera P et al: The DCR protein TTC3 affects differentiation and Golgi compactness in neurons through specific actin-regulating pathways. PloS One 2014; 9: e93721.

25 Lochhead PA, Sibbet G, Morrice N, Cleghon V: Activation-loop autophosphorylation is mediated by a novel transitional intermediate form of DYRKs. Cell 2005; 121 : 925-936.

26 Lepagnol-Bestel AM, Zvara A, Maussion G et al: DYRK1A interacts with the REST/ NRSF-SWI/SNF chromatin remodelling complex to deregulate gene clusters involved in the neuronal phenotypic traits of Down syndrome. Hum mol Genet 2009; 18: 1405-1414.

27 Abekhoukh S, Planque C, Ripoll C et al: Dyrk1A, a serine/threonine kinase, is involved in ERK and Akt activation in the brain of hyperhomocysteinemic mice. Mol Neurobiol 2013; 47: 105-116.

28 Boku S, Nakagawa S, Takamura N et al: GDNF facilitates differentiation of the adult dentate gyrus-derived neural precursor cells into astrocytes via STAT3. Biochem Biophys Res Commun 2013; 434: 779-784.

29 McMillan EL, Kamps AL, Lake SS, Svendsen CN, Bhattacharyya A: Gene expression changes in the MAPK pathway in both Fragile $\mathrm{X}$ and Down syndrome human neural progenitor cells. Am J Stem Cells 2012; 1: 154-162.

30 Kelly PA, Rahmani Z: DYRK1A enhances the mitogen-activated protein kinase cascade in PC12 cells by forming a complex with Ras, B-Raf, and MEK1. Mol Biol Cell 2005; 16: 3562-3573

31 Lee Y, Ha J, Kim HJ et al: Negative feedback Inhibition of NFATc1 by DYRK1A regulates bone homeostasis. J Biol Chem 2009; 284: 33343-33351.

32 Walte A, Ruben K, Birner-Gruenberger R et al: Mechanism of dual specificity kinase activity of DYRK1A. FEBS J 2013; 280: 4495-4511.

33 Lund IV, Hu Y, Raol YH et al: BDNF selectively regulates GABAA receptor transcription by activation of the JAK/STAT pathway. Sci Signal 2008; 1: ra9.

34 Chen CK, Bregere C, Paluch J, Lu JF, Dickman DK, Chang KT: Activity-dependent facilitation of Synaptojanin and synaptic vesicle recycling by the Minibrain kinase. Nat Commun 2014; 5: 4246.

35 Tejedor F, Zhu XR, Kaltenbach $\mathrm{E}$ et al: minibrain: a new protein kinase family involved in postembryonic neurogenesis in Drosophila. Neuron 1995; 14: 287-301.

36 Laguna A, Barallobre MJ, Marchena MA et al: Triplication of DYRK1A causes retinal structural and functional alterations in Down syndrome. Hum Mol Genet 2013; 22 : 2775-2784.

37 Rachdi L, Kariyawasam D, Guez F et al: Dyrk1a haploinsufficiency induces diabetes in mice through decreased pancreatic beta cell mass. Diabetologia 2014; 57: 960-969.

38 Hong SH, Lee KS, Kwak SJ et al: Minibrain/Dyrk1a regulates food intake through the Sir2-FOXO-sNPF/NPY pathway in Drosophila and mammals. PLoS Genet 2012; 8: e1002857.

39 Thevenon J, Callier P, Thauvin-Robinet $C$ et al: De Novo 21q22.1q22.2 deletion including RUNX1 mimicking a congenital infection. Am J Med Genet A 2011; 155A: 126-129.

40 Izumi K, Brooks SS, Feret HA, Zackai EH: $1.9 \mathrm{Mb}$ microdeletion of 21q22.11 within Braddock-Carey contiguous gene deletion syndrome region: dissecting the phenotype. Am J Med Genet A 2012; 158A: 1535-1541.

41 Lee H, Deignan JL, Dorrani N et al: Clinical exome sequencing for genetic identification of rare Mendelian disorders. JAMA 2014; 312: 1880-1887.

42 Wapner RJ, Martin CL, Levy B et al: Chromosomal microarray versus karyotyping for prenatal diagnosis. N Engl J Med 2012; 367: 2175-2184.

43 Souchet B, Guedj F, Sahun I et al: Excitation/inhibition balance and learning are modified by Dyrkla gene dosage. Neurobiol Dis 2014; 69: 65-75.

44 De la Torre R, De Sola S, Pons M et al: Epigallocatechin-3-gallate, a DYRK1A inhibitor, rescues cognitive deficits in Down syndrome mouse models and in humans. Mol Nutr Food Res 2014; 58: 278-288.

45 Guedj F, Bianchi DW, Delabar JM: Prenatal treatment of Down syndrome: a reality? Curr Opin Obstet Gynecol 2014; 26: 92-103.

Supplementary Information accompanies this paper on European Journal of Human Genetics website (http://www.nature.com/ejhg) 